\title{
Temperature Dependent Dynamic Mechanical Properties of Magnetorheological Elastomers: Experiment and Modeling
}

\author{
Yanxiang Wan ${ }^{1, a}$, Yeping Xiong ${ }^{1, b}$, Shenming Zhang ${ }^{2, c}$ \\ ${ }^{1}$ University of Southampton, UK \\ ${ }^{2}$ Lloyd's Register, UK \\ ayw8g12@soton.ac.uk, bY.Xiong@soton.ac.uk, ’Shengming.Zhang@lr.org \\ *Yanxiang Wan
}

\begin{abstract}
Magnetorheological elastomers (MREs) are a group of smart composite materials which are composed of magnetic particles dispersed in an elastomeric matrix. The controllable dynamic properties of these materials rely on many factors, in which temperature is a significant influencing factor requiring further investigations. In this paper, the dynamic mechanical analysis (DMA) tests have been performed to determine the viscoelastic properties of MREs with different test conditions. Based on the experiment results, the dynamic properties of MREs is modelled respectively by fractional Maxwell model (FMM) and generalized Maxwell model (GMM), and then the master curve of complex modulus is constructed using the time-temperature superposition (TTS) principle. The results show that the transition behavior of the silicon rubber based MRE samples under uniaxial compression occurs at about $50^{\circ} \mathrm{C}$. The storage modulus exhibits two different trends with the temperature variation: It first decreases rapidly and then increases slightly or maintains a stable value with increasing temperature.
\end{abstract}

Key words: Magnetorheological elastomer, temperature effect, dynamic mechanical analysis test, viscoelasic modeling, master curve.

\section{Introduction}

Magnetorheological elastomers (MREs) are a group of smart composite materials which are composed of micron-sized ferromagnetic particles dispersed in an elastomeric matrix. The spatial distribution of the ferromagnetic powders in the elastomer can be either homogenous (isotropic) in the absence of external magnetic field or chain-like columnar (anisotropic)in the presence of external magnetic field during curing process. The viscoelastic properties of MREs normally varied instantly and reversibly because of the dipole interaction in the presence of magnetic field. In most cases, anisotropic MREs appear to be more sensitive to the applied magnetic field with slightly larger magnetorheological (MR) effect than isotropic MREs [1, 2]. The material modulus rises with the increasing magnetic flux intensity until the magnetic saturation. The higher volume fraction of magnetic particles produces a higher MR effect, with an optimal volume fraction around 30\% leading to the maximum of both MR effect and the long-term stability of MREs [3-5]. 
The controllable dynamic properties of MREs have attracted more and more attentions, gaining wide application prospects in dynamic vibration control, such as MRE based active-adaptive tuned vibration absorber and semi-active/passive variable stiffness and damping isolator [6-8]. MRE based devices are often operated in the wide range of load frequency and amplitude, magnetic field, and temperature. With respect to design and practical application, it is essential to investigate and model the dependent dynamic properties of MREs under various loading conditions. Among these influence factors, the temperature is one of most important factors and needs to be studied. The temperature changes in MREs and MRE based devices may be caused by the environmental temperature variations or/and internal temperature rise due to energy dissipation. The shear storage modulus of cis-polybutadiene (BR) rubber based MRE samples decreased linearly with the increasing test temperature, while the shear storage modulus of natural rubber (NR) based MRE samples first increased with the increment of temperature and then decreased when the testing temperature is elevated [9]. The internal temperature of the MRE isolator was generated by the electromagnetic coil and had a remarkable effect on the stiffness and damping of the MRE isolator [10].

In small amplitude excitation, the linear viscoelastic model may applied to describe the elastic and rheological properties of MREs under harmonic loads. Li et al. [11] proposed a four-parameter linear viscoelastic model by connecting the classical three-parameter standard viscoelastic solid model in parallel with a nonlinear spring representing the magnetic field induced stiffness. Zhu et al. [12] presented another type of four-parameter linear viscoelastic model which is composed of the fractional Kelvin-Voigt model, a dashpot, and a magnetic field dependent spring in parallel. Norouzi et al. [13] developed a modified Kelvin-Voigt model to describe the nonlinear relationships between shear stress and shear strain of MREs under external magnetic field. The magnetic field dependent parameters in the proposed model were expressed by polynomial functions.

In order to investigate the effect of temperature on dynamic mechanical properties of MREs, the DMA tests have been performed for anisotropic MRE samples expose to vary temperatures and magnetic fields. Based on the achieved experiment results, the FMM and GMM are presented to describe the linear viscoelastic properties of MREs under uniaxial compression, after which the master curve of complex modulus is constructed using the time-temperature superposition principle. The model parameters are identified by minimizing the difference between the experimental data and predicted results of both storage modulus and loss modulus. The quality of the curve fit is discussed in detail by statistical analysis.

\section{Experimental setup}

The anisotropic MRE samples consisted of the silicone rubber (Wacker Chemie AG, Germany) 
and micron-sized iron particles (Sigma-Aldrich, US). The diameter of iron particles is 5 to $9 \mu \mathrm{m}$ $\geq 99.5 \%$. The synthesis of the MRE samples consist of three steps: Firstly, Elastosil A and Elastosil B were dispensed in a volume fraction of 10:1 and then the iron powders in a volume fraction of $30 \%$ were added. Secondly, the mixture was well blended and placed in a vacuum chamber for 20 min to reduce the air bubbles trapped inside the material during mixing progress. Finally, the mixture was put into the aluminum moulds and cured for 20 hours under room temperature with an external magnetic field of about $300 \mathrm{mT}$ produced by a pair of the cylindrical grade $\mathrm{N} 42$ neodymium permanent magnets (E-magnets, UK).
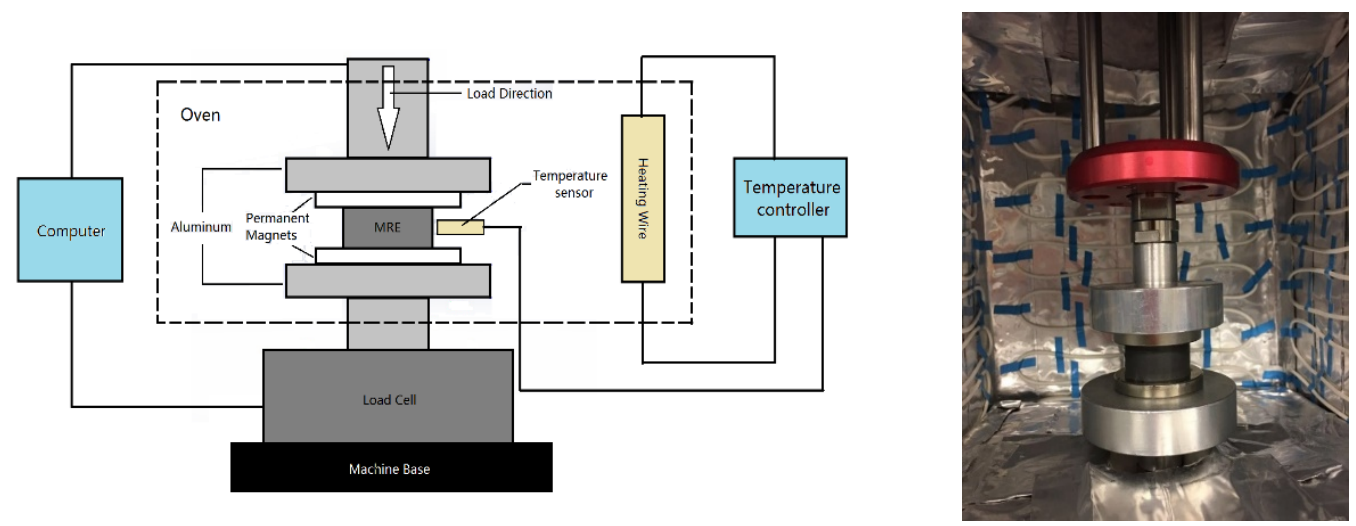

Figure 1: Experiment setup for DMA test of MRE sample under uniaxial compression

The DMA tests of the MRE samples were performed according to the BS ISO 4664-1:2011 by using Instron test machine (E1000 Electro plus) in the Transport Systems Research Laboratory. The influence of external magnetic field and test temperature on the dynamic properties of the MRE samples was achieved by a self-manufactured oven and a pair of permanent magnets as shown in Figure 1. The oven consists of a thermo-detector, the aluminum shell with thermal insulation material, and the heating wire and its control panel. During DMA test, the MRE specimen temperature was monitored and controlled within the desired temperature range. The magnetic field was produced by pairs of the cylindrical grade N42 neodymium permanent magnets (E-magnets, UK) and the uniaxial harmonic compression strain was applied and controlled by Instron test machine (E1000 Electro plus).

The material properties of the samples were measured through DMA tests in a frequency range from 0 to $60 \mathrm{~Hz}$, strain amplitudes from 0 to 1.5\%, the temperatures from room temperature (about $25^{\circ} \mathrm{C}$ ) till $60^{\circ} \mathrm{C}$, and magnetic field intensities from 0 to $500 \mathrm{mT}$. Each set of tests was carried out with three pairs of MRE samples independently and every test was repeated twice for each pair of MRE samples.

\section{Viscoelastic models}


The linear viscoelastic relationships between stress and strain can be described by the fractional Maxwell element which consists of a spring and a springpot in series. The constitutive equation of the fractional Maxwell element can be expressed in the following form [14].

$$
\sigma+\tau^{\alpha} \frac{d^{\alpha} \sigma}{d t^{\alpha}}=E \tau^{\alpha} \frac{d^{\alpha} \varepsilon}{d t^{\alpha}} \quad 0<\alpha<1
$$

in which $E$ is the spring stiffness, $\eta$ is the springpot viscosity, $\tau=\eta / E$ denotes relaxation time of fractional Maxwell element, and $\alpha$ is a fractional derivative. The springpot changes to a linear spring for the extreme case of $\alpha=0$, and leads to the dashpot in the extreme case of $\alpha=1$.

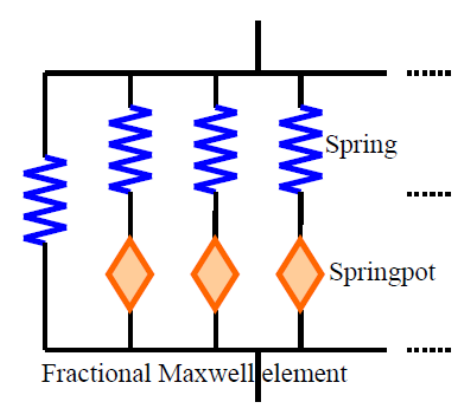

(a)

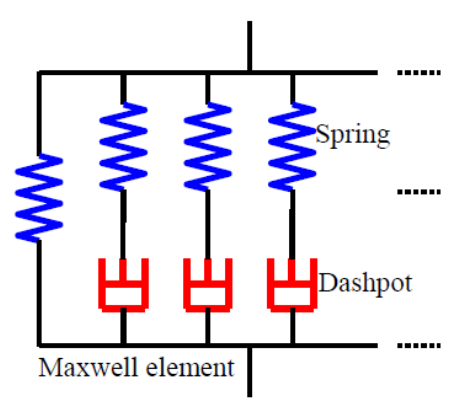

(b)

Figure 2: Linear viscoelastic models: (a) FMM and (b) GMM

The complex modulus of the fractional Maxwell element can be expressed as follow by turning Equation (1) into the frequency domain.

$$
E^{*}(i \omega)=\frac{\sigma_{0}^{*}(i \omega)}{\varepsilon_{0}^{*}(i \omega)}=\frac{E(i \omega \tau)^{\alpha}}{1+(i \omega \tau)^{\alpha}}
$$

In order to obtain a good description of the linear viscoelastic behavior in a wide range of frequencies and time scales, the fractional Maxwell model (FMM) is often adopted which comprised of a spring and several fractional Maxwell elements in parallel as shown in Figure 2(a). The complex modulus of the FMM can be obtained by summing the complex modulus of a spring and each fractional Maxwell element defined in Equation (2).

$$
\begin{aligned}
& E^{*}(i \omega)=E^{\prime}(\omega)+i E^{\prime \prime}(\omega) \\
& E^{\prime}(\omega)=E_{0}+\sum_{j=1}^{m} E_{j} \frac{\left(\omega \tau_{j}\right)^{2 \alpha_{j}}+\left(\omega \tau_{j}\right) \cos \frac{\alpha_{j} \pi}{2}}{1+\left(\omega \tau_{j}\right)^{2 \alpha_{j}}+2\left(\omega \tau_{j}\right)^{\alpha_{j}} \cos \frac{\alpha_{j} \pi}{2}}
\end{aligned}
$$




$$
E "(\omega)=\sum_{j=1}^{m} \frac{E_{j}\left(\omega \tau_{j}\right)^{\alpha_{j}} \sin \frac{\alpha_{j} \pi}{2}}{1+\left(\omega \tau_{j}\right)^{2 \alpha_{j}}+2\left(\omega \tau_{j}\right)^{\alpha_{j}} \cos \frac{\alpha_{j} \pi}{2}}
$$

where $m$ is the number of fractional Maxwell elements, the model parameters: $E_{0}, E_{j}$, $\tau_{j}$, and $\alpha_{j}$ are dependent on magnetic field and temperature, which can be identified according to the DMA test results.

For the extreme case of $\alpha_{j}=1$, the fractional Maxwell elements in FMM in Figure 2(a) are replaced by the Maxwell elements in the generalized Maxwell model (GMM) as shown in Figure 2(b). The storage modulus and loss modulus of the GMM in Figure 2(b) can be obtained by recalling Equation (4) and (5) in the extreme case of $\alpha_{j}=1$.

$$
\begin{aligned}
& E^{\prime}(\omega)=E_{0}+\sum_{j=1}^{m} E_{j} \frac{\left(\omega \tau_{j}\right)^{2}}{1+\left(\omega \tau_{j}\right)^{2}} \\
& E^{\prime \prime}(\omega)=\sum_{j=1}^{m} \frac{E_{j}\left(\omega \tau_{j}\right)}{1+\left(\omega \tau_{j}\right)^{2}}
\end{aligned}
$$

\section{Results and discussion}

\subsection{Hysteresis loops}

Figure 3 shows the influence of magnetic field and temperature on the shape of the stress-strain hysteresis loop of MRE samples under uniaxial compression. The area enclosed by the stress-strain hysteresis loop indicates the dissipated energy in each cycle and the slope of the hysteresis loop characterizes the magnitude of the complex modulus. Each of the stress-strain hysteresis loops as shown in Figure 3 approaches an elliptical shape. The little deviation in the vicinity of strain peaks which may be caused by the interfacial friction due to a change in applied strain direction. 


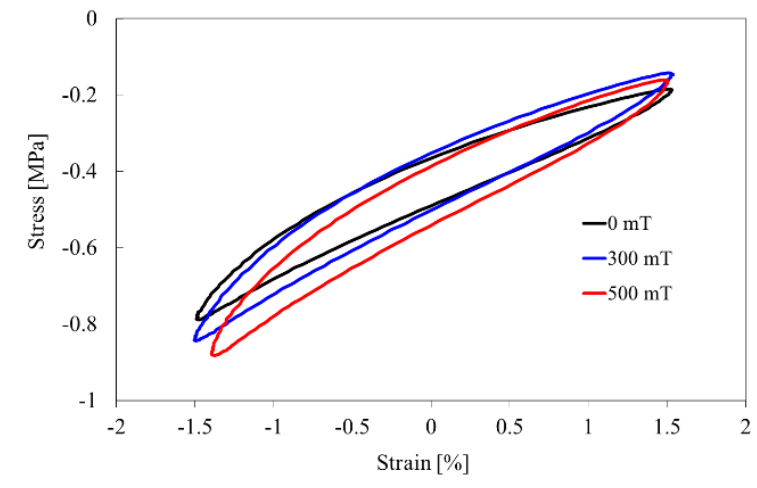

(a)

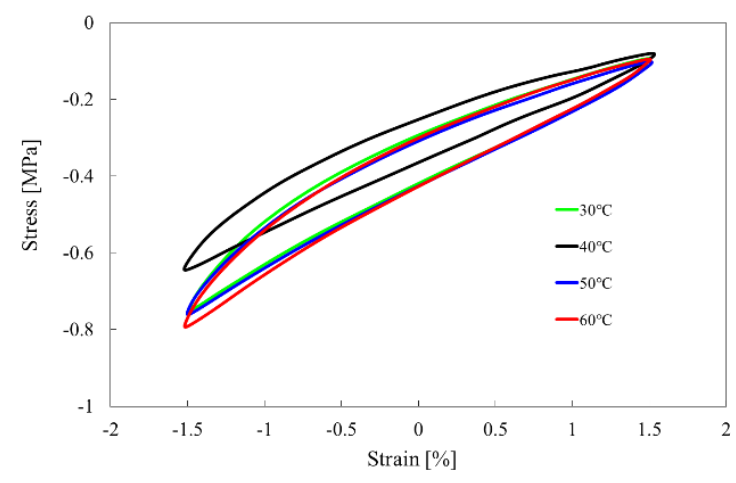

(b)

Figure 3: Dependence of hysteresis loops on: (a) magnetic field, and (b) temperature

Figure 3(a) illustrates the magnetic field dependent stress-strain hysteresis loops of the MRE samples which were measured under room temperature (about $25^{\circ} \mathrm{C}$ ) and uniaxial harmonic compression strain with a amplitude of $1.5 \%$ and a frequency of $10 \mathrm{~Hz}$. It can noticed from Figure 3(a) that the slope of the stress-strain hysteresis loop increases as the magnetic flux intensity increases, which means the material become stiffer when increasing of magnetic field strength. The temperature dependent stress-strain hysteresis loops of the MRE samples as shown in Figure 3(b) were measured under an external magnetic field of $300 \mathrm{mT}$ and uniaxial harmonic compression with a strain amplitude of $1 \%$ and a frequency of $60 \mathrm{~Hz}$. It is found from Figure 3(b) that there is a transition behavior of the MRE samples under uniaxial compression for the slope of stress-strain hysteresis loop indicating a change in the stiffness of the MRE samples. It first decreases with increasing temperature until the transition temperature and then increases or maintains constant with the increase of temperature up to $60^{\circ} \mathrm{C}$. The transition behavior occurs at about $50^{\circ} \mathrm{C}$ is mainly related to the alpha phase transition of the silicon rubber matrix and the variation of dipole interaction of magnetic particle due to temperature changes.

\subsection{Dependent dynamic moduli}

Figure 4 shows the influence of temperature on the dynamic moduli of the MRE under magnetic field of $300 \mathrm{mT}$ and $500 \mathrm{mT}$ respectively. The experimental data reveals that the vatiation of the storage modulus with temperature can be mainly divided into two stages within a environment temperature ranging from room temperature to $60^{\circ} \mathrm{C}$. The storage modulus first decreases rapidly with the increment of temperature and then starts to increase slightly or maintains a stable value with further increasing temperature. 

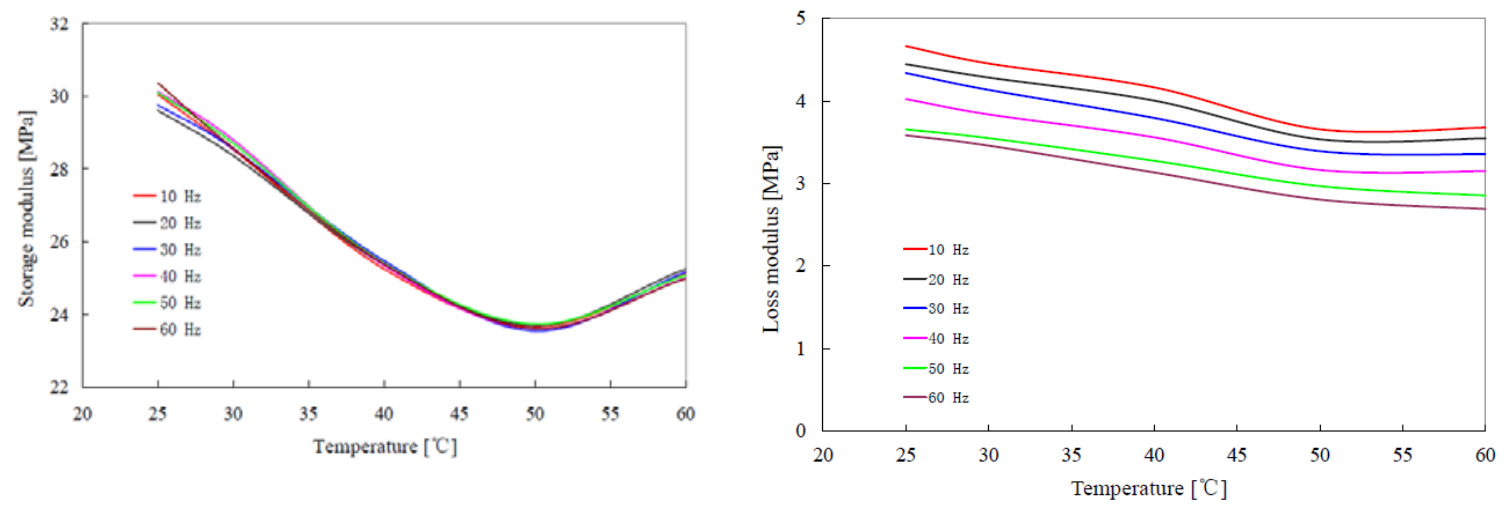

(a)
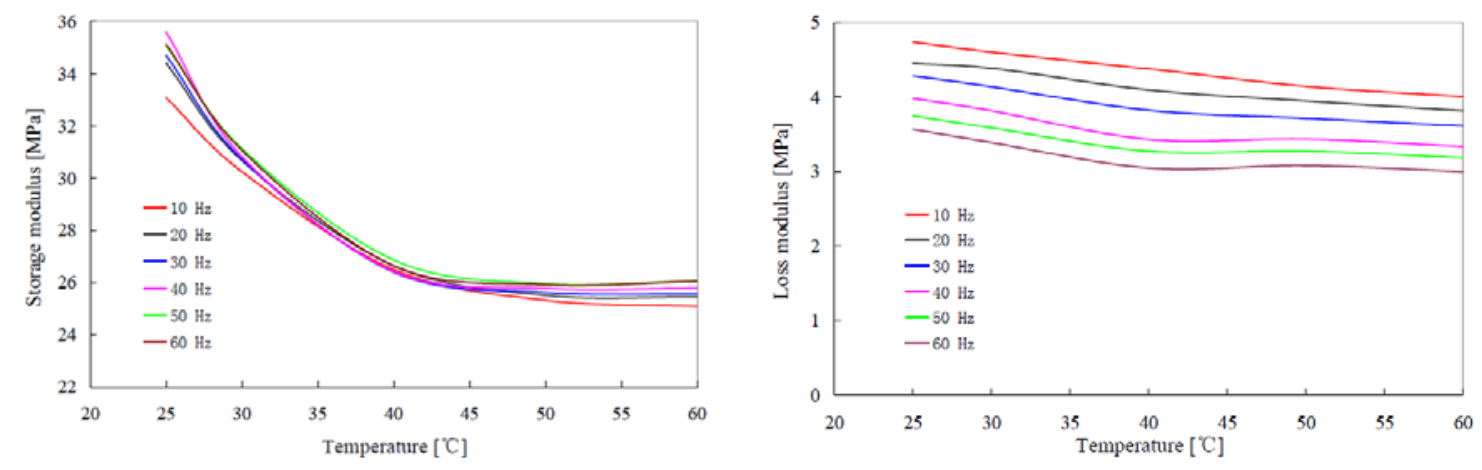

(b)

Figure 4: Dependence of storage and loss moduli on frequency and temperature under strain amplitude of 1\%, and magnetic field of: (a) $300 \mathrm{mT}$, and (b) $500 \mathrm{mT}$

According to Figure 4, the tendency of the storage modulus with the temperature under different magnetic fields is similar below the transition temperature but differentiate the transition temperature. Figure 4(a) shows that the storage modulus of the MRE samples under magnetic field of $300 \mathrm{mT}$ decreases rapidly with the increasing of temperature and reaches the minimum value at the temperature of $50^{\circ} \mathrm{C}$, which is $21 \%$ lower than the room temperature (about $25^{\circ} \mathrm{C}$ ) condition. Then the storage modulus starts to increase again as the temperature increases until $60^{\circ} \mathrm{C}$. Figure 4(b) reveals that the storage modulus of the MRE samples under magnetic field of $500 \mathrm{mT}$ decreases rapidly with the increasing temperature and has the minimum value at the temperature of $50^{\circ} \mathrm{C}$, it is about 25\% lower than the room temperature condition. When the temperature keeps rising, the storage modulus begins to increase slightly with ascending temperature but the increment is small and almost constant until $60{ }^{\circ} \mathrm{C}$. However the relationship between loss modulus changes and temperature variation is different. The loss modulus decreases linearly from the maximum value below the transition temperature and then approximately maintains constant with increasing temperature until $60^{\circ} \mathrm{C}$. 
The dynamic modulus of MREs can be divided into mechanical and magnetic parts. The mechanical part represents the dynamic modulus of particle reinforced elastomer and depends on the excitation frequency and temperature in a linear viscoelastic range. The magnetic part represents magnetic field-induced dynamic modulus of iron particle reinforced elastomer and depends on the mechanical modulus and MR effect. Therefore the transition temperature appears at about $50^{\circ} \mathrm{C}$ may be regarded as a result of the superposition of two factors: the alpha phase transition of the particle reinforced silicone rubber [9], as well as the interaction between iron particles under the magnetic field when the silicon rubber matrix become soft due to the temperature elevation. According to differential scanning calorimetry (DSC) thermal analysis result, the transition behavior begin at about $50^{\circ} \mathrm{C}$ and reaches the exothermic peaks at $58.66^{\circ} \mathrm{C}$. This indicates that the silicon rubber matrix starts the alpha phase transition at about $50^{\circ} \mathrm{C}$. The difference between Figure 4(a) and Figure 4(b) reveals that the MR effect is more sensitive to temperature in a higher magnetic field.

\subsection{Comparison between experimental results and modeling predictions}

The model parameters of the FMM and GMM are dependent on temperature and magnetic field and can be identified respectively by using the genetic algorithm (GA) in Matlab global optimization toolbox to minimizing the sum of square of error (SSE) between the experiment data and prediction results of both storage and loss moduli. Figure 5 presents the comparison between the dynamic moduli obtained by DMA tests and predicted by using the FMM and GMM. It can be found that the FMM fit better for both storage and loss moduli than the GMM. The fitting results can be evaluated in detail by using the criteria of the goodness-of-fit The standard error ratio $\left(\mathrm{S}_{\mathrm{e}} / \mathrm{S}_{\mathrm{y}}\right)$ and the coefficient of determination $\left(\mathrm{R}^{2}\right)$ are expressed respectively in the statistical analysis as follows [15],

$$
\begin{aligned}
& S_{e}=\sqrt{\frac{\sum\left(y_{\exp }-y_{\text {pred }}\right)^{2}}{n-k}} \\
& S_{y}=\sqrt{\frac{\sum\left(y_{\exp }-\bar{y}_{\text {exp }}\right)^{2}}{n-1}} \\
& R^{2}=1-\frac{n-k}{n-1}\left(\frac{S_{e}}{S_{y}}\right)^{2}
\end{aligned}
$$

where, $n$ and $k$ are the number of the samples and the independent model parameters respectively, $y_{\text {exp }}$ and $y_{\text {pred }}$ are the experimental data and predicted results of the sample respectively, and $y_{\exp }$ bar denotes the mean value of the experimental data, e.g. experimental data of storage modulus or loss modulus. 
The comparison of the standard error ratio and the coefficient of determination between FMM and GMM are listed in Table 1. As the model parameters increasing from four to seven, the excellent (marked with red color in Table 1) or good (marked with blue color in Table 1) fitting results were obtained by using FMM according to the criteria of the fitting performance statistical analysis [15]. While the fitting result of the GMM is not good and the improvement is not obvious when simply adding $\mathrm{k}$.
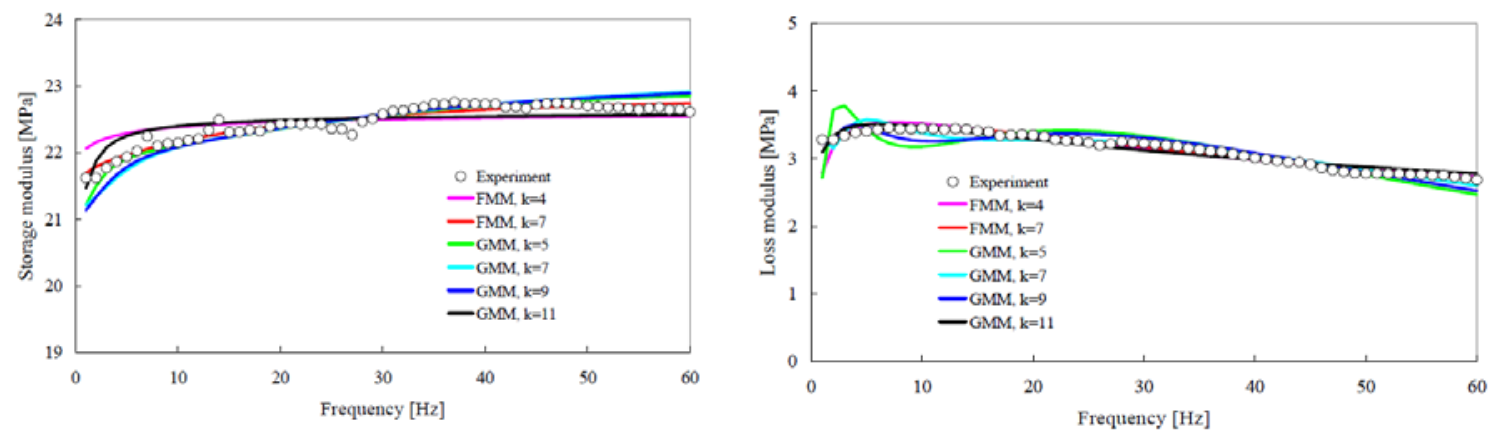

(a)
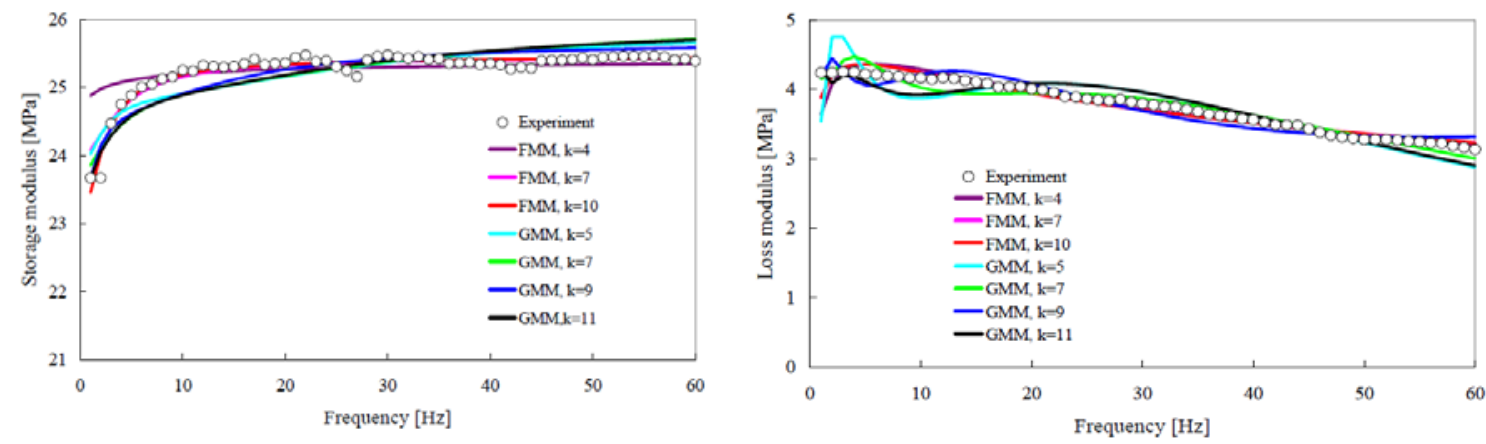

(b)
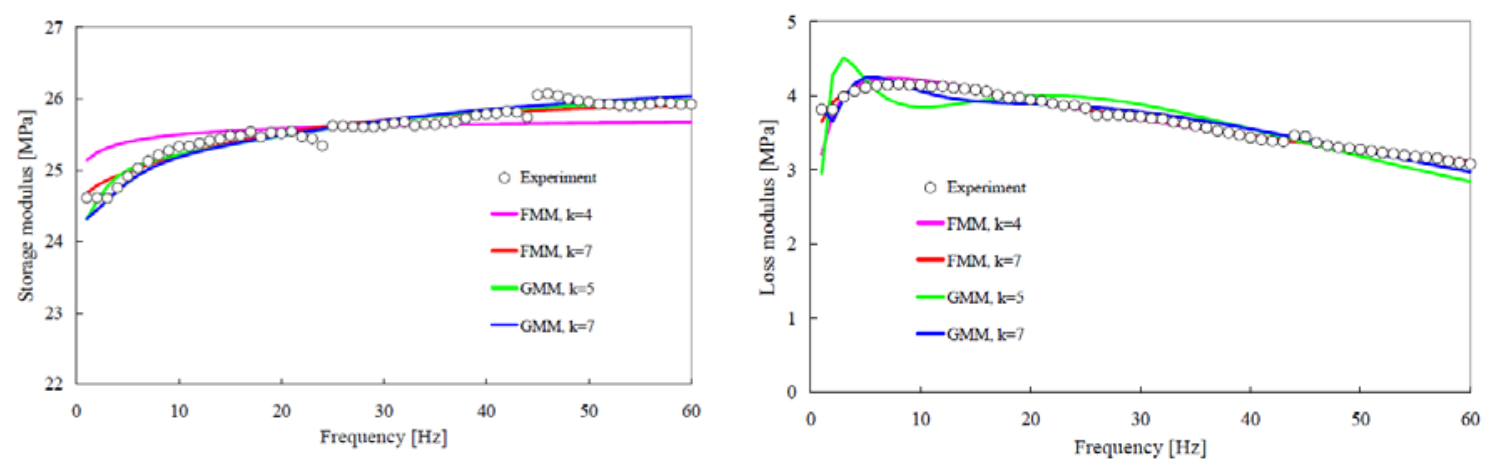

(c)

Figure 5: Comparison between measured data and predicted results of storage and loss moduli under: (a) $0 \mathrm{mT}$ and $30^{\circ} \mathrm{C}$, (b) $300 \mathrm{mT}$ and $40^{\circ} \mathrm{C}$, and (c) $500 \mathrm{mT}$ and $50^{\circ} \mathrm{C}$

Table 1: Comparison of the goodness-of-fit between FMM and GMM

\begin{tabular}{|c|c|c|c|}
\hline Case & $0 \mathrm{mT}$ and $30^{\circ} \mathrm{C}$ & $300 \mathrm{mT}$ and $40^{\circ} \mathrm{C}$ & $500 \mathrm{mT}$ and $50^{\circ} \mathrm{C}$ \\
\hline
\end{tabular}




\begin{tabular}{|c|c|c|c|c|c|c|c|}
\hline \multicolumn{2}{|c|}{ Dynamic moduli } & $\mathrm{E}^{\prime}(\mathrm{MPa})$ & E” (MPa) & $\overline{E^{\prime}(\mathrm{MPa})}$ & E” (MPa) & $\mathrm{E}^{\prime}(\mathrm{MPa})$ & E” (MPa) \\
\hline \multirow{2}{*}{$\begin{array}{c}\text { FMM, } \\
\mathrm{k}=4\end{array}$} & $\mathrm{~S}_{\mathrm{e}} / \mathrm{S}_{\mathrm{y}}$ & 0.70 & 0.34 & 0.78 & 0.30 & 0.74 & 0.27 \\
\hline & $\mathrm{R}^{2}$ & 0.54 & 0.89 & 0.42 & 0.92 & 0.49 & 0.93 \\
\hline \multirow{2}{*}{$\begin{array}{c}\text { FMM, } \\
k=7\end{array}$} & $\mathrm{~S}_{\mathrm{e}} / \mathrm{S}_{\mathrm{y}}$ & 0.31 & 0.14 & 0.36 & 0.25 & 0.30 & 0.11 \\
\hline & $\mathrm{R}^{2}$ & 0.92 & 0.98 & 0.88 & 0.95 & 0.92 & 0.99 \\
\hline \multirow{2}{*}{$\begin{array}{c}\text { FMM, } \\
k=10\end{array}$} & $\mathrm{~S}_{\mathrm{e}} / \mathrm{S}_{\mathrm{y}}$ & 1 & I & 0.26 & 0.25 & I & I \\
\hline & $\mathrm{R}^{2}$ & I & I & 0.94 & 0.94 & l & l \\
\hline \multirow{2}{*}{$\begin{array}{c}\text { GMM, } \\
\mathrm{k}=5\end{array}$} & $\mathrm{~S}_{\mathrm{e}} / \mathrm{S}_{\mathrm{y}}$ & 0.42 & 0.71 & 0.65 & 0.58 & 0.29 & 0.65 \\
\hline & $\mathrm{R}^{2}$ & 0.83 & 0.53 & 0.60 & 0.68 & 0.93 & 0.61 \\
\hline \multirow{2}{*}{$\begin{array}{c}\text { GMM, } \\
\mathrm{k}=7\end{array}$} & $\mathrm{~S}_{\mathrm{e}} / \mathrm{S}_{\mathrm{y}}$ & 0.56 & 0.30 & 0.70 & 0.30 & 0.31 & 0.26 \\
\hline & $\mathrm{R}^{2}$ & 0.72 & 0.92 & 0.55 & 0.92 & 0.91 & 0.94 \\
\hline \multirow{2}{*}{$\begin{array}{c}\text { GMM, } \\
\mathrm{k}=9\end{array}$} & $\mathrm{~S}_{\mathrm{e}} / \mathrm{S}_{\mathrm{y}}$ & 0.55 & 0.43 & 0.58 & 0.30 & I & 1 \\
\hline & $\mathrm{R}^{2}$ & 0.74 & 0.84 & 0.70 & 0.92 & 1 & 1 \\
\hline
\end{tabular}

The temperature and frequency dependent dynamic modulus of thermorheologically simple materials at different temperatures can be shifted into a master curve according to the timetemperature superposition (TTS) principle [15-17]. Recently, the application of master curve based on TTS has been extended to describe MR fluid [18] and the multi-phase materials where both components have the similar temperature dependence [19]. The complex modulus function can be described in terms of a single master curve and shift factors as follow:

$$
E^{*}(\omega, T)=b_{T} E^{*}\left(a_{T} \omega, T_{r e f}\right)
$$

where $\mathrm{a}_{\mathrm{T}}$ and $\mathrm{b}_{\mathrm{T}}$ are the horizontal and vertical shift factors respectively, and $\mathrm{T}_{\text {ref }}$ is a reference temperature.

Once the complex modulus of the MRE samples is obtained as shown in Figure 6(a), the reference temperature is selected as $25^{\circ} \mathrm{C}$ in this study. The measured data at all the other temperatures are merged into a single master curve as shown in Figure 6(b) by using the unrestricted shift factors. Little discrepancy is observed in Figure 6(b) and the goodness-of-fit parameters, $\mathrm{S}_{\mathrm{e}} / \mathrm{S}_{\mathrm{y}}=0.134$ and $\mathrm{R}^{2}=0.983$, indicating an excellent correlation between measured data and predicted results of the constructed master curve. 


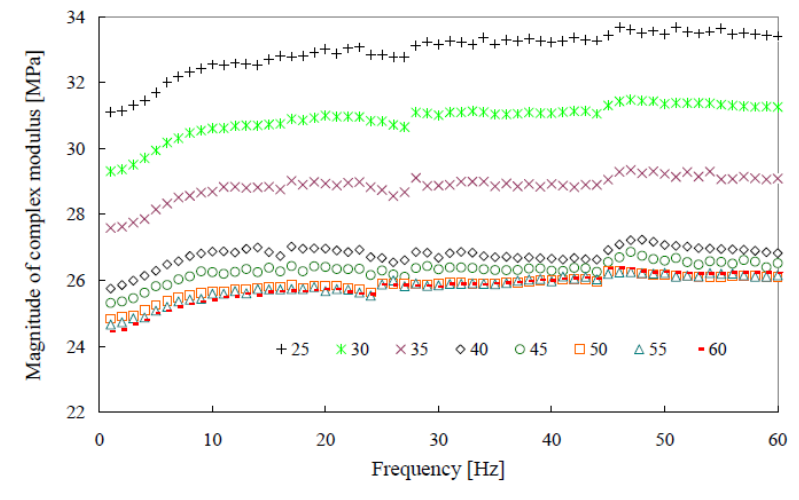

(a)

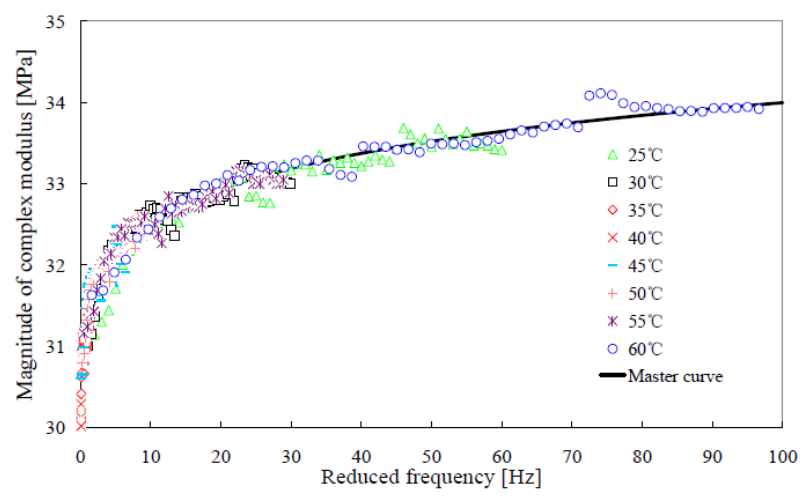

(b)

Figure 6: Temperature dependent magnitude of complex modulus (a) and the constructed master curve at a reference temperature of $25^{\circ} \mathrm{C}(\mathrm{b})$

\section{Conclusion}

The experimental and modeling studies have benn performed on the dynamic properties of the anisotropic MRE samples under different magnetic fields and test temperatures. The experimental results show that the transition temperature of the MRE samples appears at about $50^{\circ} \mathrm{C}$ The storage modulus exhibits two different trends below and above the transition temperature:It first decreases rapidly with temperature increased, and then and then increases slightly or maintains a stable value with increasing temperature until $60^{\circ} \mathrm{C}$

The FMM and GMM were presented respectively to model the temperature and frequency dependent dynamic modulus of the anisotropic MRE samples in uniaxial compression. TTS was employed to shift the isotherms of dynamic modulus into the master curve at a reference temperature of $25^{\circ} \mathrm{C}$. The excellent correlation between the experimental data and modeling resultswas confirmed by the goodness-of-fit statistical analysis.

\section{Reference}

[1] H. Böse and R. Röder, "Magnetorheological elastomers with high variability of their mechanical properties”, J. Phys. Conf. Ser. 149, 012090 (2009).

[2] J. Kaleta, M. Królewicz, D. Lewandowski, “Magnetomechanical properties of anisotropic and isotropic magnetorheological composites with thermoplastic elastomer matrices”, Smart Mater. Struct. 20, 085006 (2011).

[3] L.C. Davis, “Model of magnetorheological elastomers”, J. Appl. Phys. 85, 3348 (1999).

[4] W.H. Li and X.Z. Zhang, “A study of the magnetorheological effect of bimodal particle based magnetorheological elastomers”, Smart Mater. Struct. 19, 035002 (2010). 
[5] Y.F. Zhou, S. Jerrams, A. Betts, G. Farrell, L. Chen, "The influence of particle content on the equi-biaxial fatigue behaviour of magnetorheological elastomers”, Mater. Des. 67(15), 398-404 (2015).

[6] G.J. Liao, X.L. Gong, C.J. Kang, S.H. Xuan, “The design of an active-adaptive tuned vibration absorber based on magnetorheological elastomer and its vibration attenuation performance”, Smart Mater. Struct. 20, 075015 (2011).

[7] M. Behrooz, X.J. Wang, F. Gordaninejad, “Control of structures featuring a new MRE isolator system”, Proc. of SPIE 8341 (2012).

[8] C. Collette, G. Kroll, G. Saive, V. Guillemier, M. Avraam, A. Preumont, “ Isolation and damping properties of magnetorheologic elastomers”, J. Phys. Conf. Ser. 149, 012091 (2009).

[9] W. Zhang, X.L. Gong, S.H. Xuan, W.Q. Jiang, “Temperature-dependent mechanical properties and model of magnetorheological elastomers”, Ind. Eng. Chem. Res. 50, 6704-6712 (2011).

[10] M. Yu, L.J. Zhao, J. Fu, M. Zhu, "Thermal effects on the laminated magnetorheological elastomer isolator”, Smart Mater. Struct. 25, 115039 (2016).

[11] W.H. Li, Y. Zhou, T.F. Tian, "Viscoelastic properties of MR elastomers under harmonic loading”, Rheol. Acta, 49, 733-740 (2010).

[12] J.T. Zhu, Z.D. Xu, Y.Q. Guo. "Magnetoviscoelasticity parametric model of an MR elastomer vibration mitigation device”, Smart Mater. Struct. 21, 075034 (2012).

[13] M. Norouzi, S.M.S. Alehashem, H. Vatandoost, Y.Q. Ni, M.M. Shahmardan, “A new approach for modeling of magnetorheological elastomers”, J. Intell. Mater. Syst. Struct. 27(8), 1121-1135 (2016).

[14] M. Sasso, G. Palmieri, D. Amodio, "Application of fractional derivative models in linear viscoelastic problems”, Mech. Time-Depend. Mater. 15, 367-387 (2011).

[15] N.I.M. Yusoff, E. Chailleux, G.D. Airey, “A comparative study of the influence of shift factor equations on master curve construction”, Int. J. Pav. Res. Technol. 4, 324-336, (2011).

[16] W.M. Madigosky, “A method for modeling polymer viscoelastic data and the temperature shift function”, J. Acoust. Soc. Am. 119(6), 3760-3765 (2006).

[17] N.W. Tschorgl, W.G. Kbauss, I. Emrischoegl, "The effect of temperature and pressure on the mechanical properties of thermo- and/or piezorheologically simple polymeric materials in thermodynamic equilibrium - A critical review”, Mech. Time-Depend. Mater. 6, 53-99 (2002).

[18] W.W. Chooi and S.O. Oyadiji, "Characterizing the effect of temperature and magnetic field strengths on the complex shear modulus properties of magnetorheological (MR) fluid”, Int. J. Modern Phys. B 19, 1318-1324 (2005).

[19] T. Nakano, “Applicability condition of time-temperature superposition principle (TTSP) to a multi-phase system”, Mech. Time-Depend. Mater. (2012). 